\title{
Thermal properties and flame retardancy of an ether-type UV-cured polyurethane coating
}

\author{
L. J. Chen ${ }^{1,2}$, Q. L. Tai ${ }^{1,3}$, L. Song ${ }^{1}$, W. Y. Xing ${ }^{1}$, G. X. Jie ${ }^{4}, Y . H u^{1,4 *}$
}

${ }^{1}$ State Key Laboratory of Fire Science, University of Science and Technology of China, 96 Jinzhai Road, Hefei, Anhui 230026, P.R. China

2Department of Polymer Science and Engineering, University of Science and Technology of China, 96 Jinzhai Road, Hefei, Anhui 230026, P.R. China

${ }^{3}$ Suzhou Institute for Advanced Study, University of Science and Technology of China, 166 Ren'ai Road, Suzhou, Jiangsu 215123, P.R. China

${ }^{4}$ State Key Laboratory of Environmental Adaptability for Industrial Products, China National Electric Apparatus Research Institute, Guangzhou, 510300, P.R. China

Received 28 March 2010; accepted in revised form 19 May 2010

\begin{abstract}
A new UV-reactive monomer piperazine-N,N'-bis(acryloxyethylaryl-phosphoramidate) (N-PBAAP) containing phosphorus and nitrogen was synthesized and used as flame retardant for an ether-type UV-cured polyurethane acrylate (PUA) coating. The thermal properties of the PUA films were investigated by thermogravimetric analysis (TGA) in air and nitrogen atmosphere. The TGA results showed that the incorporation of N-PBAAP can obviously enhance the char residue of the PUA coatings. From the TGA and real time Fourier transformed infrared spectroscopy (RT-FTIR) results, different degradation behaviors were observed in the PUA coatings with different N-PBAAP content. The combustibility of the PUA coatings was evaluated by microscale combustion calorimeter (MCC). The MCC results revealed that the addition of NPBAAP in the coatings can significantly reduce the peak Heat Release Rate (pHRR), Heat Release Capacity (HRC) and the Total Heat Release (THR) of the samples. Furthermore, dynamical mechanical thermal analysis (DMA) was employed to examine the viscoelastic properties of the PUA films. It was found that the incorporation of N-PBAAP in the formulation can bring in more functional groups to the coatings, which results in an increase of the glass transition temperature $\left(T_{g}\right)$ and cross linking density (XLD) of the films.
\end{abstract}

Keywords: coatings, flame retardancy, polyurethane acrylate, thermal properties

\section{Introduction}

It is well known that the conventional solventbased coatings will cause environmental pollution during their thermal curing processes by emitting a large amount of volatile organic compounds (VOC) and other hazardous air pollutants (HAP). The radiation curing process has been found to be an effective alternative to solvent borne technology in the coating industry [1]. Radiation curing technology, mostly UV curing, offers many advantages during the curing process, in relation to the ecology or environment, energy conservation, economics and in the excellent performance of products [2-4]. The most commonly used UV-curable formulations involve unsaturated acrylic oligomers, including epoxy acrylates, polyester acrylates, polyether acrylates, urethane acrylates and silicone acrylates [5-8]. Among the oligomers used for UV-curable coatings, urethane acrylate oligomers offer a wide range of excellent application properties, such as high impact and tensile strength, abrasion resistance and toughness etc. As a result, UV-curable 
urethane coatings have found a large number of applications on various substrates such as wood, plastics, metal, glass and ceramics [9-11].

In addition, PU coatings are often used as 'passive fireproofing materials', which refers to insulating systems designed to decrease heat transfer from a fire to the structure being protected. These are usually known as 'intumescent coatings', and can be described as follows: When the temperature of the coating surface reaches a critical temperature under the heat of the fire, the surface begins to decompose and is converted into a highly viscous liquid. At the same time, reactions are initiated that result in the release of inert gases with low thermal conductivity. These gases are trapped inside the viscous fluid leading to the formation of bubbles. The result is the expansion or foaming of the coating, sometimes up to several times its original thickness, to form a protective carbonaceous char that serves as an insulating barrier between the fire and the substrate. In fact, phosphorus-nitrogen (P-N) synergism, i.e. intumescent flame-retardant system, is recognized in many literatures [12-18]. In the P-N system, the phosphorus-containing parts act as an acid source, they usually break down at low temperature and take part in the formation process of the carbonaceous char. On the other hand, nitrogen-containing parts are usually used as gas source, they produce incombustible gases without toxic smoke and fog during heating. The evolving gases can dilute the concentration of the oxygen near the flame and cause the char to swell and hence provide an insulating multi-cellular protective layer. This shield limits at the same time the heat transfer from the heat source to the substrate and the mass transfer from the substrate to the heat source resulting in a conservation of the underlying material.

In this work, a new UV-curable intumescent flame retardant piperazine- $\mathrm{N}, \mathrm{N}^{\prime}$-bis(acryloxyethylarylphosphoramidate) (N-PBAAP) with high phosphorus content (10.4 wt\%) was synthesized combining phosphorus and nitrogen element into one molecule. N-PBAAP was then used as a UV-reactive monomer blending with urethane acrylate oligomer (UA) in different ratios. The thermal and combustion properties of the PUA films were investigated by TG and MCC, respectively. RT-FTIR was employed to investigate the changes of chemical structures of these samples during their thermal degradation, in an attempt to elucidate the degradation mechanism. Furthermore, dynamical mechanical thermal properties were also investigated.

\section{Experimental section}

\subsection{Materials}

Phenyl dichlorophosphate (PDCP) was purchased from Deheng Chemical Co. (Shijiazhuang, China), and was freshly distilled before use. Triethylamine (TEA) and hydroxyethyl acrylate (HEA) were offered by China Medicine (Group) Shanghai Chemical Reagent Co. (Shanghai, China), and they were purified by distillation. Piperazine anhydrous was purchased from Sinopharm Chemical Reagent Co. Ltd. 2-Hydroxy-2-methyl-1-phenyl-1-propanone (Darocur 1173), kindly supplied by Ciba-Geigy, Switzerland, was used as a photoinitiator. UA, which is an ether-type urethane diacrylate oligomer with $15 \%$ TPGDA (Tripropylene glycol diacrylate) as dilute monomer, was supplied by Wuxi Tianjiaosaite Co. Tetrahydrofuran (THF) using as solvent was refluxed with sodium, and was then distilled before use.

\subsection{Synthesis of N-PBAAP}

PDCP $(0.1 \mathrm{~mol}, 21.1 \mathrm{~g})$ was placed into a threenecked flask in an ice-bath equipped with a mechanical stirrer and a dropping funnel. Triethylamine $(0.21 \mathrm{~mol}, 21.2 \mathrm{~g})$ was added by the funnel under stirring. HEA $(0.1 \mathrm{~mol}, 11.6 \mathrm{~g})$ was diluted with THF (20 ml) and was slowly dropped into the above reaction vessel within 2 hours. The mixture was then kept stirring for 4 hours. After that piperazine anhydrous $(0.05 \mathrm{~mol}, 4.307 \mathrm{~g})$ dissolved in dry THF $(90 \mathrm{ml})$ was added dropwise over a period of 2 hours. Removal of precipitated triethylamine hydrochloride by filtering and evaporation of filtrate under a reduced pressure gave the crude product. The product was purified further by washing it with hydrochloric acid, aqueous solutions of $5 \mathrm{wt} \%$ sodium bicarbonate and saturated sodium chloride, respectively. Finally, a salmon pink viscous liquid was obtained (79.2\% yield). The success of synthesis was confirmed by ${ }^{1} \mathrm{H}$ NMR and ${ }^{31} \mathrm{P}$ NMR analysis.

${ }^{1} \mathrm{H} \mathrm{NMR}\left(\mathrm{CDCl}_{3}\right): \delta \quad(\mathrm{ppm})=7.16 \sim 7.32 \quad($ aromatic $\mathrm{H}), 5.84 \sim 5.87\left(\mathrm{CH}=\mathrm{CH}_{2}\right.$,trans $), 6.08 \sim 6.15$ 
$\left(\mathrm{CH}=\mathrm{CH}_{2}, \mathrm{cis}\right), 6.41 \sim 6.46\left(\mathrm{CH}=\mathrm{CH}_{2}\right), 4.27 \sim 4.39$ $\left(-\mathrm{OCH}_{2} \mathrm{CH}_{2} \mathrm{O}-\right), 3.13 \sim 3.15\left(\mathrm{~N}\left(\mathrm{CH}_{2}-\right)_{2}\right) ;{ }^{31} \mathrm{P}$ NMR $\left(\mathrm{CDCl}_{3}\right): \delta(\mathrm{ppm})=4.38$.

FTIR $\left(\mathrm{KBr}, \mathrm{cm}^{-1}\right): 1263(-\mathrm{P}=\mathrm{O}), 1165,979$ $(-\mathrm{P}-\mathrm{O}-\mathrm{C}), 1726(-\mathrm{C}=\mathrm{O}), 1640,1410,812(-\mathrm{C}=\mathrm{C}-)$, 930, $769(-\mathrm{P}-\mathrm{N}-\mathrm{C})$.

\subsection{Sample preparation}

The mixtures of UA with N-PBAAP in different ratios were stirred thoroughly to get various homogenous blends. UA, and UA/N-PBAAP blends were then poured into a mold sized $25 \times 25 \mathrm{~mm}^{2}$, and the thickness of the cured film is about $1 \mathrm{~mm}$. Later the resin blends with $3 \mathrm{wt} \%$ Darocur 1173 were exposed to a UV irradiation equipment $\left(80 \mathrm{~mW} \cdot \mathrm{cm}^{-2}\right.$, made by Lantian Co., China), which emits light in the near UV (characterized wavelength, 340 360 nm) in air atmosphere. The distance between the sample and UV lamp is $10 \mathrm{~cm}$, and the exposure time is about $15 \mathrm{~s}$. The structures of N-PBAAP, UA oligomer and the sample preparation process were shown in Figure 1.

\subsection{Characterization and analysis}

\subsubsection{FTIR analysis}

The FTIR spectra were recorded with MAGNA-IR 750 spectrometer (Nicolet Instrument Company, USA, part of Thermo Fisher Scientific) using $\mathrm{KBr}$ pellet. The transmission mode was used and the wavenumber range was set from 4000 to $400 \mathrm{~cm}^{-1}$. Real time Fourier transform infrared (RT-FTIR) method was employed to study the thermo-oxidative degradation of the N-PBAAP/PUA cured films. Granule of the cured sample was mixed with
$\mathrm{KBr}$ powder, and the mixture was pressed into a tablet, which was then placed into the oven. The temperature of the oven was raised at a heating rate of about $10^{\circ} \mathrm{C} / \mathrm{min}$. RT-FTIR spectra were collected during the thermo-oxidative degradation of the cured samples.

\subsection{2. ${ }^{1} \mathrm{H}$ NMR and ${ }^{31} \mathrm{P}$ NMR analysis}

${ }^{1} \mathrm{H}$ NMR and ${ }^{31} \mathrm{P}$ NMR was performed on a AVANCE 400 Bruker spectrometer (Bruker Corporation, Billerica, MA, USA) at room temperature using chloroform-D as a solvent.

\subsubsection{Thermogravimetric analysis (TGA)}

The thermogravimetric analysis was carried out on a TGA Q5000 IR thermalgravimetric analyzer (TA Instruments, New Castle, Delaware, USA). About $5 \mathrm{mg}$ of cured samples were put in ceramic crucibles and heated from 30 to $700^{\circ} \mathrm{C}$ at a heating rate of $20^{\circ} \mathrm{C} \cdot \mathrm{min}^{-1}$ under air and nitrogen purge, respectively (flow rate of $100 \mathrm{ml} / \mathrm{min}$ ).

\subsubsection{Microscale combustion calorimeter (MCC)}

The MCC tests were carried out on a Govmark MCC-2 Microscale Combustion Calorimeter (Govmark, Farmingdale, NY, USA), which is a pyrolysis-combustion flow calorimeter. In the test, 4 $6 \mathrm{mg}$ powdery cured samples were heated to $650^{\circ} \mathrm{C}$ at a heating rate of $1 \mathrm{~K} / \mathrm{s}$ in an inert gas steam (nitrogen, $80 \mathrm{ml} \cdot \mathrm{min}^{-1}$ ). Then the volatile was mixed with oxygen $\left(20 \mathrm{ml} \cdot \mathrm{min}^{-1}\right)$ prior to entering a $900^{\circ} \mathrm{C}$ combustion furnace and the heat of com-

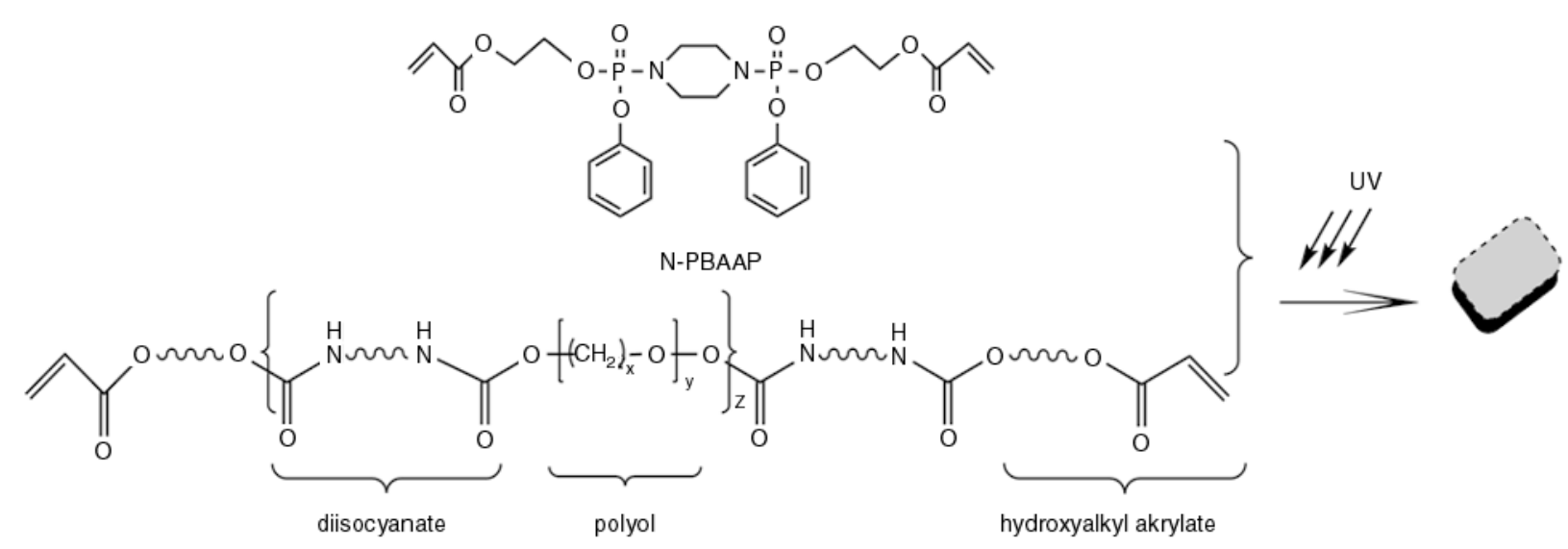

Figure 1. Preparation of PUA/N-PBAAP films 
bustion of the pyrolysis products was measured by oxygen consumption principle.

\subsubsection{DMA test}

The tensile storage modulus $\left(E^{\prime}\right)$ and tensile loss factors $(\tan \delta)$ of the cured films were measured by a dynamic mechanical thermal analyzer (Diamond DMA, PerkinElmer Co., Waltham, MA, USA) at a frequency of $10 \mathrm{~Hz}$ and a heating rate of $10^{\circ} \mathrm{C} \cdot \mathrm{min}^{-1}$ in the range of -75 to $125^{\circ} \mathrm{C}$ with $25 \times 5 \times 1 \mathrm{~mm}^{3}$ specimens. The crosslink density $(X L D)$ as the molar number of elastically effective network chain per cube centimeter of the film, was calculated from the storage modulus in the rubbery plateau region according to Equation (1):

$$
X L D=\frac{E^{\prime}}{3 R T}
$$

where $E^{\prime}$ is the elastic storage modulus, $R$ is the ideal gas constant, and $T$ is the temperature in $\mathrm{K}$.
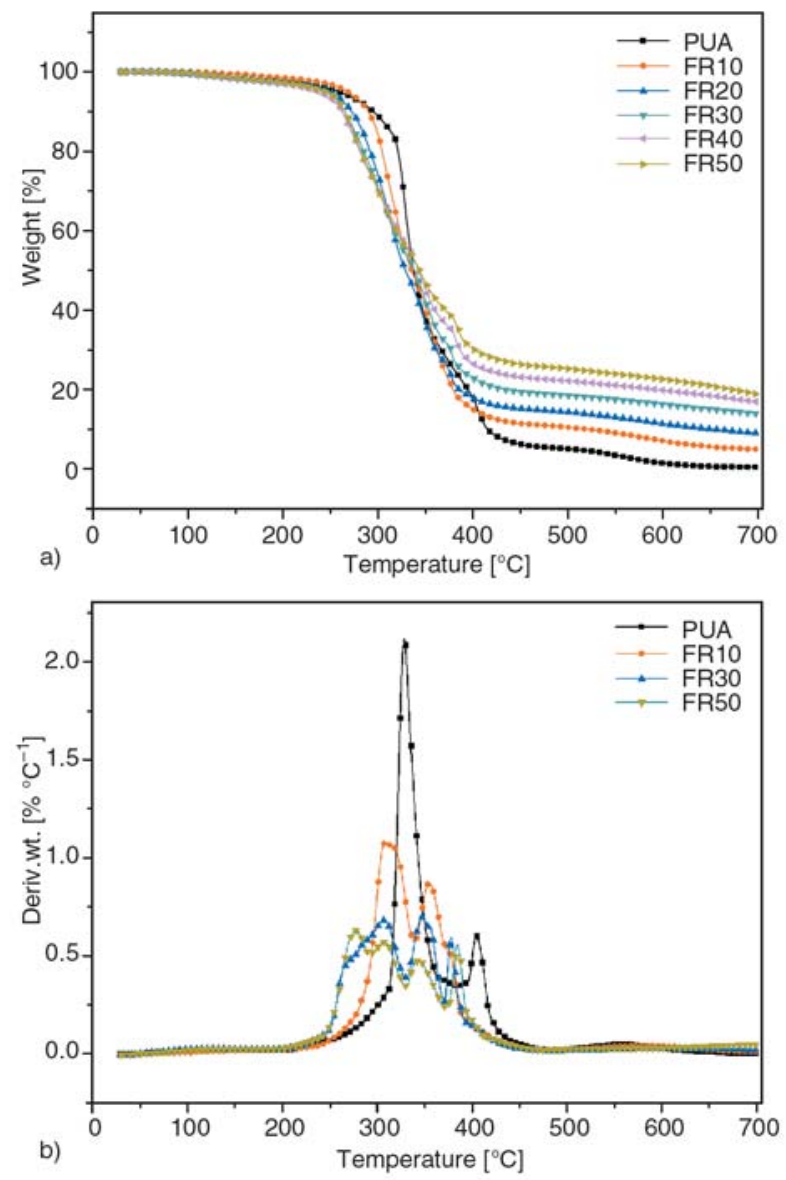

Figure 2. (a) TG curves (b) DTG curves of PUA and PUA/N-PBAAP cured films in air

\section{Results and discussion}

\subsection{Thermogravimetric analysis}

Thermogravimetric analysis (TGA) was used to investigate the effect of N-PBAAP on the thermal stability of the PUA coatings. Figure $2 \mathrm{a}$ and Figure $3 \mathrm{a}$ show the TG curves of PUA and PUA/ N-PBAAP cured films in air and nitrogen atmosphere; Figure $2 b$ and Figure $3 b$ show the DTG curves of PUA and PUA films with 10, 30, $50 \mathrm{wt} \%$ N-PBAAP loadings. The details of the TGA data for the coating compositions in air and nitrogen are presented in Tables 1 and 2, respectively, showing the temperatures for different percentage weight losses and the percentage char residues at different temperatures of the coatings.

From Figure 2a, it can be seen that, in air atmosphere, all the PUA/N-PBAAP films have more char residues than the pure PUA film at high temperature regions. As can be seen from Figure $b$, the pure PUA film undergoes a major weight loss in the temperature range of $250-360^{\circ} \mathrm{C}$ due to thermal
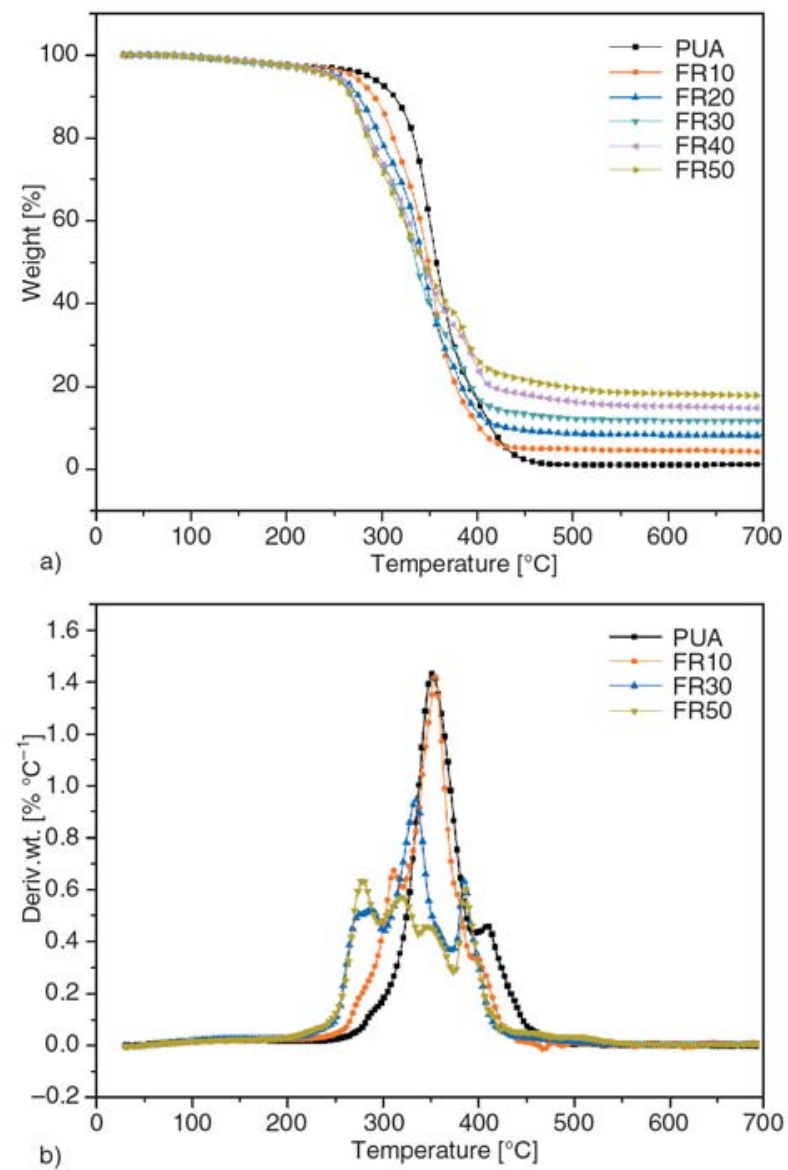

Figure 3. (a) TG curves (b) DTG curves of PUA and PUA/N-PBAAP cured films in nitrogen 
Table 1. Details of the TGA data for the coating compositions in air

\begin{tabular}{|c|c|c|c|c|c|c|c|c|}
\hline \multirow{2}{*}{ Sample name } & \multirow{2}{*}{$\begin{array}{c}\text { PUA } \\
{[w t \%]}\end{array}$} & \multirow{2}{*}{$\begin{array}{c}\text { N-PBAAP } \\
\text { [wt\%] }\end{array}$} & \multicolumn{3}{|c|}{ Temperature $\left[{ }^{\circ} \mathrm{C}\right] / \mathrm{wt}$. loss } & \multicolumn{3}{|c|}{ Char residue $[w t \%] /{ }^{\circ} \mathrm{C}$} \\
\hline & & & $10 \%$ & $30 \%$ & $50 \%$ & 500 & 600 & 700 \\
\hline PUA & 100 & 0 & 295 & 327 & 338 & 5.0 & 1.4 & 0.5 \\
\hline FR10 & 90 & 10 & 290 & 314 & 336 & 10.5 & 7.0 & 4.9 \\
\hline FR20 & 80 & 20 & 272 & 305 & 329 & 14.3 & 11.4 & 9.1 \\
\hline FR30 & 70 & 30 & 265 & 302 & 339 & 18.6 & 16.3 & 14.0 \\
\hline FR40 & 60 & 40 & 262 & 301 & 343 & 22.2 & 19.8 & 16.9 \\
\hline FR50 & 50 & 50 & 265 & 301 & 344 & 25.2 & 22.6 & 18.8 \\
\hline
\end{tabular}

Table 2. Details of the TGA data for the coating compositions in nitrogen

\begin{tabular}{|l|c|c|c|c|c|c|c|c|}
\hline \multirow{2}{*}{ Sample name } & $\begin{array}{c}\text { PUA } \\
{[\mathbf{w t} \%]}\end{array}$ & \multirow{2}{*}{$\begin{array}{c}\text { N-PBAAP } \\
{[\mathbf{w t} \%]}\end{array}$} & \multicolumn{2}{|c|}{ Temperature $\left[{ }^{\circ} \mathbf{C}\right] / \mathbf{w t}$. loss } & \multicolumn{3}{|c|}{ Char residue $[\mathbf{w t} \%]^{\circ} \mathbf{C}$} \\
\cline { 4 - 10 } & & $\mathbf{1 0 \%}$ & $\mathbf{3 0 \%}$ & $\mathbf{5 0 \%}$ & $\mathbf{5 0 0}$ & $\mathbf{6 0 0}$ & $\mathbf{7 0 0}$ \\
\hline PUA & 100 & 0 & 313 & 343 & 357 & 1.1 & 1.0 & 1.0 \\
\hline FR10 & 90 & 10 & 292 & 328 & 349 & 4.9 & 4.6 & 4.3 \\
\hline FR20 & 80 & 20 & 276 & 319 & 343 & 8.7 & 8.3 & 8.2 \\
\hline FR30 & 70 & 30 & 268 & 309 & 336 & 12.3 & 11.8 & 11.6 \\
\hline FR40 & 60 & 40 & 270 & 310 & 342 & 16.4 & 15.2 & 14.7 \\
\hline FR50 & 50 & 50 & 268 & 305 & 344 & 19.6 & 18.3 & 17.8 \\
\hline
\end{tabular}

decomposition of the organic coating and then a minor degradation step in the temperature range of $400-430^{\circ} \mathrm{C}$ due to the oxidation of the residue formed on thermal decomposition of the coating. The char residue of pure PUA cured film is $0.5 \%$ at $700^{\circ} \mathrm{C}$ as shown in Table 1, indicating the complete decomposition of PUA film. However, the PUA/ N-PBAAP coatings reveal different trends of thermal degradation. The initial degradation temperatures of the blends are lowered by the addition of N-PBAAP, and the DTG curves show broader peaks with weaker intensity and more degradation steps as the N-PBAAP loading increases in the blends. As presented in Figure 2b, FR50 (PUA film with $50 \mathrm{wt} \%$ N-PBAAP loading) shows a four-step thermal degradation process. The first region can be assigned to the decomposition of the phosphate, whereas the second is due to the thermal pyrolysis of side chains of the acrylate and the formation of char. The third region is attributed to the decomposition of the unstable structures in the char, and the last step may result from the oxidation of the exposed matrix as well as the further oxidation of the char residue formed before [12].

Comparing the DTG curves shown in Figure $2 b$ and Figure $3 b$, it can be seen that the pure PUA film exhibits similar thermal degradation behavior in nitrogen and in air, i.e. a major decomposition of the coating before $400^{\circ} \mathrm{C}$. But in the case of FR10 (PUA film with $10 \mathrm{wt} \%$ N-PBAAP loading), different degradation behaviors of the films are observed under different circumstances. We can see that, in air, sample FR10 shows obvious twostep degradation process, implying that the incorporation of N-PBAAP in the coating can slow down the rapid decomposition of the PUA matrix. While in nitrogen FR10 represents a major one-step degradation which is similar to that of the pure PUA film. This suggests that, in an inert atmosphere, the thermal degradation of the PUA film cannot be significantly influenced when the loading of N-PBAAP is low, which is different from that in air. However, as the loadings of N-PBAAP in the coatings increase, the cured flame retardant samples tend to degrade in the same way during heating both in air and nitrogen. Take FR50 for example, it shows a four-step degradation process in nitrogen as the same as that in air.

According to the above analysis and the data obtained from TGA, it can be concluded that the incorporation of N-PBAAP in the PUA films can obviously enhance the flame retardancy and the char residues of the coatings both in air and in nitrogen.

\subsection{Combustion properties of PUA/N-PBAAP cured films}

The Microscale Combustion Calorimeter (MCC) is one of the most effective methods for investigating the combustion properties of polymer materials. The test improves upon previous methods by directly measuring the heat of combustion of the gases evolved during controlled heating of the sam- 


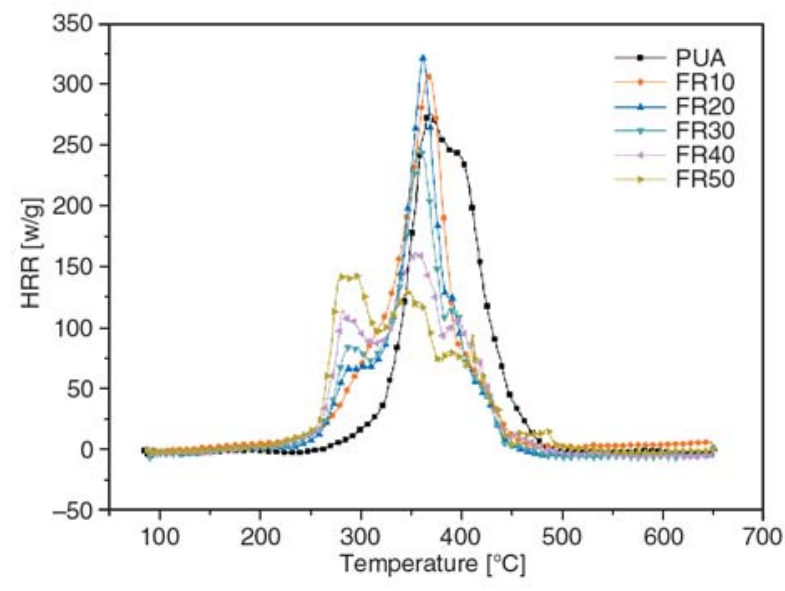

Figure 4. The heat release rate (HRR) curves of PUA, and PUA/N-PBAAP cured films

ples. As a result, the flammability parameters determined by MCC correlate with the intrinsic flammability properties of polymers and it offers a convenient methodology for estimating the fire hazard potential of a material from just a few milligrams of samples [19].

Figure 4 shows the heat release rate (HRR) curves of PUA, and PUA/N-PBAAP cured films blended in different ratios and the corresponding combustion data are presented in Table 3. It is found that the peak HRR (pHRR) of PUA blends decreases with the increase of N-PBAAP content. Compared with the pure PUA film, the pHRR of the sample containing $50 \mathrm{wt} \%$ N-PBAAP decreased from 275.3 to $142.5 \mathrm{~W} / \mathrm{g}$. On the other hand, it is observed that the films with low content of N-PBAAP (FR10 and FR20) exhibit higher pHRR than that of the pure PUA film. This may be caused by the decomposition of the weak $\mathrm{P}-\mathrm{O}-\mathrm{C}$ structure in the phosphate at low temperature, which may release combustible low-molecule-weight segments during degradation due to no oxidation in an inert atmosphere. Attributed to the same reason, the temperature at maximum pyrolysis rate $\left(T_{\max }\right)$ also decreases with the increasing N-PBAAP content. However, in view of the total heat release (THR), all blends

Table 3. Corresponding combustion data from MCC

\begin{tabular}{|l|c|c|c|c|}
\hline $\begin{array}{c}\text { Sample } \\
\text { name }\end{array}$ & $\begin{array}{c}\text { HRC } \\
{[\mathbf{J} / \mathbf{g} \cdot \mathbf{K}]}\end{array}$ & $\begin{array}{c}\mathbf{p H R R} \\
{[\mathbf{W} / \mathbf{g}]}\end{array}$ & $\begin{array}{c}\text { THR } \\
{[\mathbf{k J} / \mathbf{g}]}\end{array}$ & $\begin{array}{c}\mathbf{T}_{\max } \\
{\left[{ }^{\circ} \mathbf{C}\right]}\end{array}$ \\
\hline PUA & 279 & 275.3 & 23.0 & 368.5 \\
\hline FR10 & 300 & 303.5 & 21.0 & 366.9 \\
\hline FR20 & 318 & 321.6 & 19.1 & 361.8 \\
\hline FR30 & 247 & 249.3 & 18.7 & 358.2 \\
\hline FR40 & 164 & 161.7 & 18.5 & 354.7 \\
\hline FR50 & 142 & 142.5 & 18.6 & 294.4 \\
\hline
\end{tabular}

have lower THR than pure PUA film. The heat release capacity (HRC), which is obtained by dividing the maximum value of the specific heat release rate (SHRR) with the heating rate in the test, is a material property. HRC appears to be a good predictor of flammability and propensity for ignition, and it is related to the decomposition kinetics and combustion parameters of the sample [20]. It can be seen from Table 3 that HRC shows the similar variation of pHRR, indicating the incorporation of $\mathrm{N}$ PBAAP can effectively decrease the combustion potential of PUA. All the results above show that N-PBAAP is an effective flame retardant for PUA coatings.

\subsection{Thermal degradation mechanism}

With the purpose of elucidating the thermal degradation processes of the pure PUA and the flame retardant PUA films, three samples were selected (PUA,FR10 and FR50), and the characterization of these samples was conducted using RT-FTIR spectroscopy.

The real time FTIR observed for PUA, FR10 and FR50 at selected temperatures between RT (room temperature) and $450^{\circ} \mathrm{C}$ are shown respectively in Figure 5, Figure 6 and Figure 7. The assignments of the major bands observed at RT are summarized in Table 4 on the basis of recent IR studies of various types of polyurethanes and phosphates. The thermal degradation of polyurethanes has been reported in several studies [21,22], it is considered that the degradation of an ether-type polyurethane begins with the dissociation and thermal decompo-

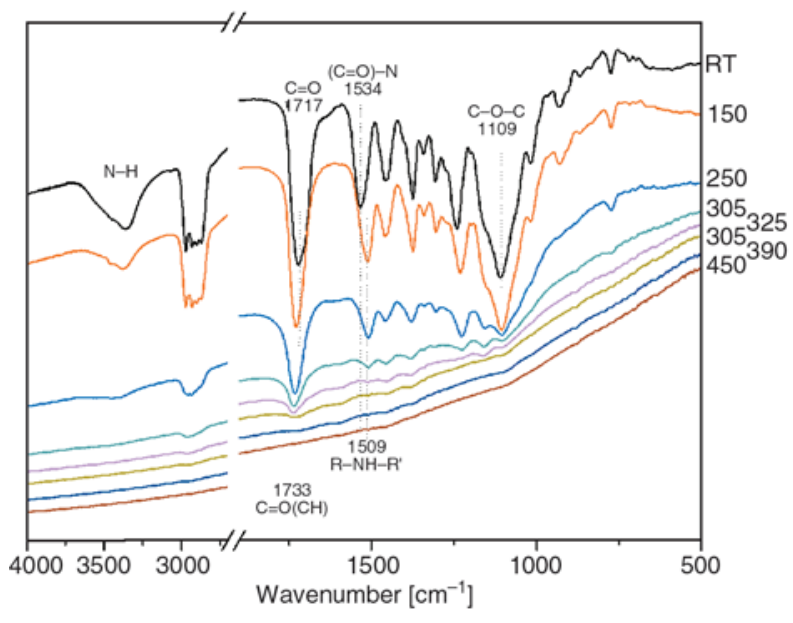

Figure 5. The real time FTIR spectra for PUA film at selected temperatures 


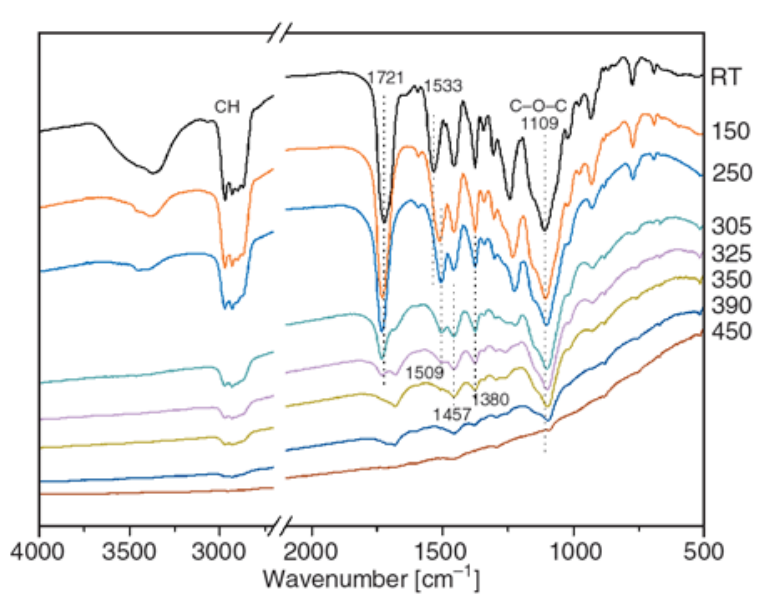

Figure 6. The real time FTIR spectra for sample FR10 at selected temperatures

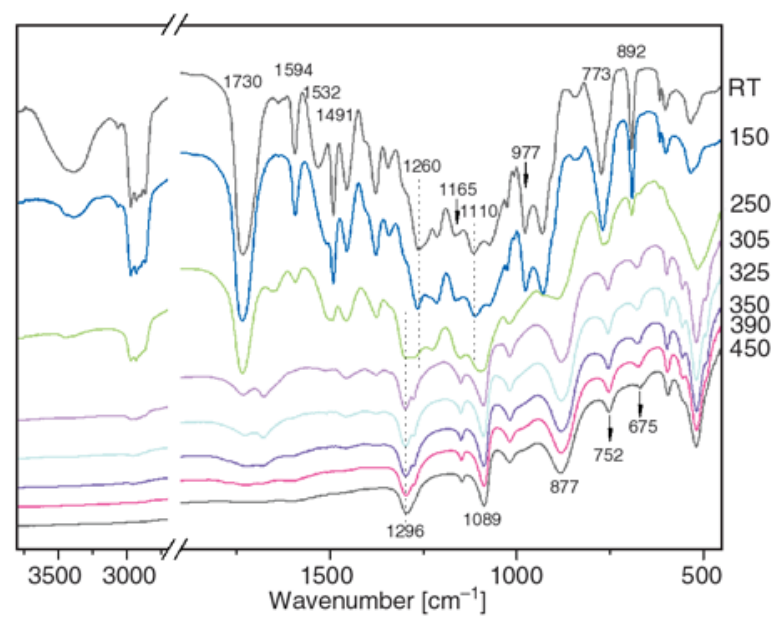

Figure 7. The real time FTIR spectra for sample FR50 at selected temperatures

Table 4. Assignments of peaks in real time FTIR of PUA, FR10 and FR50

\begin{tabular}{|l|l|}
\hline $\begin{array}{c}\text { Frequency } \\
{\left[\mathbf{c m}^{-1}\right]}\end{array}$ & \multicolumn{1}{c|}{ Assignment ${ }^{\mathrm{a}}$} \\
\hline 3480 & (vs) free urethane $\mathrm{N}-\mathrm{H}$ stretching \\
\hline 3400 & (vs) $\mathrm{N}-\mathrm{H}$ hydrogen-bonded to urethane $>\mathrm{C}=\mathrm{O}$ \\
\hline 3310 & (vs) $\mathrm{N}-\mathrm{H}$ hydrogen-bonded to ether oxygen \\
\hline 2949,2865 & (as) $\mathrm{CH}_{2}$ and (s) $\mathrm{CH}_{2}$ \\
\hline 1733 & (vs) free $\mathrm{C}=\mathrm{O}$ \\
\hline 1717 & (vs) urethane $\mathrm{C}=\mathrm{O}$ hydrogen-bonded to $\mathrm{N}-\mathrm{H}$ \\
\hline 1594,1491 & (vs) skeleton benzene ring $\mathrm{C}-\mathrm{C}$ \\
\hline 1534 & (vs) $\mathrm{C}-\mathrm{N}$ and $($ vb) $\mathrm{N}-\mathrm{H}$ of urethane \\
\hline 1509 & (vb) $\mathrm{N}-\mathrm{H}$ of polyamine \\
\hline 1457,1380 & (vb) $\mathrm{CH}$ and $\mathrm{CH}$ \\
\hline 1260 & (vs) $\mathrm{P}=\mathrm{O}$ \\
\hline 1165,977 & (vs) aromatic $\mathrm{P}-\mathrm{O}-\mathrm{C}$ \\
\hline 1110 & (as) $\mathrm{C}-\mathrm{O}-\mathrm{C}$ \\
\hline 773,692 & (vb) aromatic $\mathrm{C}-\mathrm{H}$, out of plane bend \\
\hline
\end{tabular}

a(vs): stretching vibration; (as): asymmetric stretching mode; (s): symmetric stretching mode; (vb): bending vibration

sition of the urethane hard segment. As shown in Figure 5, the intensities of the bands (around
$3350 \mathrm{~cm}^{-1}$ ) assigned to $\mathrm{N}-\mathrm{H}$ decrease intensively with increasing temperature, and almost disappear at $250^{\circ} \mathrm{C}$. At $150^{\circ} \mathrm{C}$, the peak at $1717 \mathrm{~cm}^{-1}$, which is attributed to the stretching mode of a $\mathrm{C}=\mathrm{O}$ group hydrogen-bonded to urethane $\mathrm{N}-\mathrm{H}$, is shifted to higher wave number at $1733 \mathrm{~cm}^{-1}$ (free $\mathrm{C}=\mathrm{O}$ group) [23]. These mean that the hydrogen-bond dissociation and thermal decomposition of the urethane linkage have occurred at as low temperature as $150^{\circ} \mathrm{C}$. However, as to the soft segment with ether structures, things are different. When temperature reaches $150^{\circ} \mathrm{C}$, no significant changes are observed with the characteristic bands $\left(1109 \mathrm{~cm}^{-1}\right.$ for C-O-C, 2850 2980 $\mathrm{cm}^{-1}$ for C-H) of the ether structures, indicating that the thermal stability of the ether bonds is much higher than that of the urethane linkage. As temperature increases to $250^{\circ} \mathrm{C}$, there is a large decrease in absorbance of the characteristic bands for ether structures, implying the rapid decomposition of the soft segment. But these bands are still detectable even above $325^{\circ} \mathrm{C}$. Another peak that should be noticed is the absorbance at $1534 \mathrm{~cm}^{-1}$, which is assigned to the $\mathrm{C}-\mathrm{N}$ stretching and $\mathrm{N}-\mathrm{H}$ deformation vibrations of the urethane group [8]. As can be seen at $150^{\circ} \mathrm{C}$, this peak shifts to lower wave number at $1509 \mathrm{~cm}^{-1}$, attributed to the $\mathrm{N}-\mathrm{H}$ deformation of polyamine [5]. It can be speculated that the original urethane linkage has converted into various polyamines during the degradation. All above give evidences for the thermal degradation of the ether-type PUA film in air atmosphere. Undoubtedly, the degradation process is initiated by the decomposition of the urethane group at low temperature. This step probably leads to the formation of various polyamines, which decompose later, and the release of $\mathrm{CO}_{2}$, as shown by Equation (2). Then the ether structures of the soft segment degrade intensively, releasing large amount of low-molecule-weight combustible fragments. All the bands can barely be examined at $350^{\circ} \mathrm{C}$, suggesting the full decomposition of PUA film.

$\mathrm{RNHCOOR}^{\prime} \rightarrow \mathrm{RNHR}^{\prime}+\mathrm{CO}_{2}$

The spectral region for $\mathrm{N}-\mathrm{H}$ from 3200 to $3600 \mathrm{~cm}^{-1}$ mainly involves the bands of urethane $\mathrm{N}-\mathrm{H}$ hydrogen-bonded to ether oxygen $\left(3310 \mathrm{~cm}^{-1}\right)$, of urethane $\mathrm{N}-\mathrm{H}$ hydrogen-bonded to urethane $>\mathrm{C}=\mathrm{O}\left(3400 \mathrm{~cm}^{-1}\right)$, and free urethane $\mathrm{N}-\mathrm{H}$ without hydrogen bonding $\left(3480 \mathrm{~cm}^{-1}\right)$ [23]. For FR10, as 


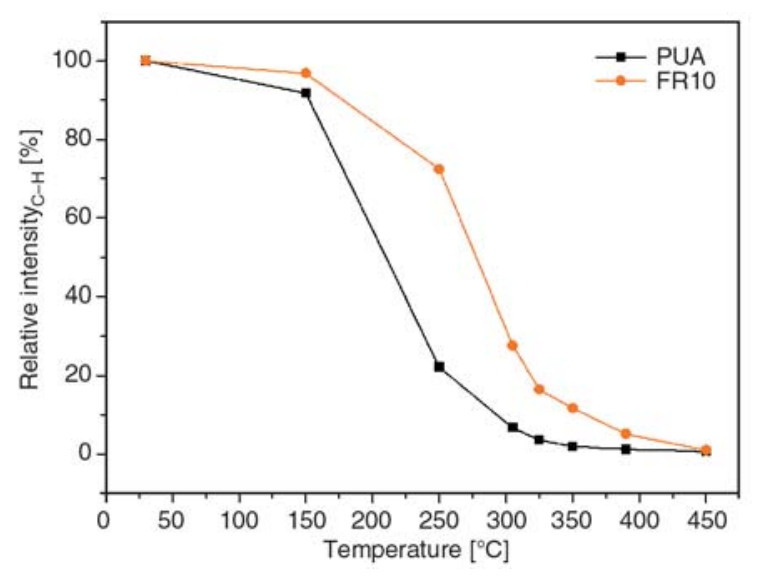

Figure 8. Relative peak intensity between 2800 and $3037 \mathrm{~cm}^{-1}$ for $\mathrm{C}-\mathrm{H}$ bond in the FTIR spectrum of UV cured PUA and FR10 films at various temperatures

shown in Figure 6, the intensities of these peaks for $\mathrm{N}-\mathrm{H}$ bond decrease rapidly as temperature increases, but they are still detected at $250^{\circ} \mathrm{C}$. Simultaneously, it is obvious that the main peak shifts to higher wave number. These indicate the hydrogen bond formed by urethane $\mathrm{N}-\mathrm{H}$ is gradually dissociated and the degradation of urethane linkage has been postponed. In view of the ether structures, peaks at $1109,1380,1457 \mathrm{~cm}^{-1}$ and peak region from 2850 to $2980 \mathrm{~cm}^{-1}$ (assigned to the vibration of soft segment shown in Table 4) are noted. Although the intensities of these peaks gradually decrease, they all remain as the temperature increases to even $390^{\circ} \mathrm{C}$. Figure 8 shows the relative intensity of the peak region between 2800 and $3037 \mathrm{~cm}^{-1}$ in the FTIR spectra of cured PUA and FR10 films for $\mathrm{C}-\mathrm{H}$ bond in $\mathrm{CH}_{2}$ and $\mathrm{CH}_{3}$ groups with temperature raising from 30 (RT) to $470^{\circ} \mathrm{C}$. It is very clear that the $\mathrm{C}-\mathrm{H}$ bond of pure PUA film decreases rapidly with increasing temperature from 150 to $250^{\circ} \mathrm{C}$, whereas the absorbance between 2800 and $3057 \mathrm{~cm}^{-1}$ for FR10 film decreases slowly at this temperature region. According to the above, it can be concluded that the degradation of the soft segment is significantly postponed by the addition of N-PBAAP with $10 \mathrm{wt} \%$ content. In other words, the addition of N-PBAAP in the coatings can delay the decomposition of the PUA matrix, and thus improve the flame retardancy of the coating.

The probable reason for the improvement of flame retardancy of the sample containing $10 \mathrm{wt} \%$ N-PBAAP can be explained as follows. We have known that the ether bond exhibits excellent ther- mal stability under an inert atmosphere [23], and the absorbance of stretching mode of $\mathrm{C}-\mathrm{O}-\mathrm{C}$ bond at $1109 \mathrm{~cm}^{-1}$ is much higher than that of other vibrational bands. During heating process, the degradation always begins with the breakage of the urethane group, releasing a lot of $\mathrm{CO}_{2}$, as has been proved above. Meanwhile, the N-PBAAP contained in the sample decomposes at a relatively low temperature around $200^{\circ} \mathrm{C}$, as is common for a phosphorus-containing flame retardant, due to the scissions of the unstable $\mathrm{P}-\mathrm{O}-\mathrm{C}$ structures. The thermal degradation of N-PBAAP, in air, leads to the formation of phosphorus-rich compounds and also the release of various kinds of incombustible gases (carbon oxide, nitrogen, and low molecular weight $\mathrm{PO}_{\mathrm{x}}$ etc.) [24]. These incombustible gases will provide a relatively inert environment for the remains of the sample, thus resulting in a delayed degradation of the matrix. However, it is difficult to confirm the formation of phosphorus-rich compounds by FTIR because of the masking effect of the broad and strong peak at $1109 \mathrm{~cm}^{-1}$ assigned to ether structure and the low content of N-PBAAP loading.

When the loading of N-PBAAP increases to $50 \mathrm{wt} \%$ in the coating, as shown in Figure 7, different thermal degradation behavior of the film is observed. The assignments of the main peaks for N-PBAAP are listed in Table 4. From Figure 7, it can be seen that the absorbance of peaks at 1165 , $977 \mathrm{~cm}^{-1}[25,26]$ and the broad peak at $3390 \mathrm{~cm}^{-1}$, which are attributed to the stretching bands of $\mathrm{P}-\mathrm{O}-\mathrm{C}$ and $\mathrm{N}-\mathrm{H}$ structures respectively, almost disappear at $250^{\circ} \mathrm{C}$. These offer information that the initial degradation of FR50 involves the scission of the urethane linkage as well as the decomposition of a large amount of $\mathrm{P}-\mathrm{O}-\mathrm{C}$ structures of N-PBAAP. Meanwhile, the changes of several peaks are examined with increasing temperature. The peak for the $\mathrm{P}=\mathrm{O}$ bond $\left(1263 \mathrm{~cm}^{-1}\right)$ is shifted to higher wave number at $1294 \mathrm{~cm}^{-1}$, which is assigned to the $\mathrm{P}=\mathrm{O}$ stretching vibration of $\mathrm{P}-\mathrm{O}-\Phi$ structure, where $\Phi$ represents the graphite-like polynuclear aromatic structure $[16,27]$. As the temperature elevates to $305^{\circ} \mathrm{C}$, the bands of 773 and $692 \mathrm{~cm}^{-1}$ are shifted to lower wave numbers at 752 and $675 \mathrm{~cm}^{-1}$, attributed to out-of-plane bending vibrations of aromatic $\mathrm{C}-\mathrm{H}$ for $\mathrm{P}-\mathrm{O}-\Phi$ complex structures [16, 28]. In addition, new peaks at 1089 and $877 \mathrm{~cm}^{-1}$ assigned to symmetrical and 
asymmetrical stretching vibrations of $\mathrm{P}-\mathrm{O}-\mathrm{P}$ structure also appear at elevated temperatures [29]. All these changes of the peaks in the spectra give significant evidences that the original phosphate structure of N-PBAAP has been converted into various kinds of $\mathrm{P}-\mathrm{O}-\mathrm{P}$ and $\mathrm{P}-\mathrm{O}-\Phi$ complex structures when temperature elevates to $305^{\circ} \mathrm{C}$. However, the characteristic peaks for the ether structures are barely detected at $305^{\circ} \mathrm{C}$. According to the above analysis, it can be concluded that a phosphorus-rich layer is formed on the surface of the PUA matrix due to the decomposition of N-PBAAP at low temperature. Combining with the gases released, it is believed that the intumescent char layer covering the surface of the matrix can serve as a heat barrier and protect the matrix from the heat and fire, thus resulting in an enhancement of the flame retardancy and a high char yield of the coatings.

In conclusion, according to the results obtained from the RT-FTIR, we have proposed different degradation mechanisms for the selected samples. The pure PUA film first decomposes from the urethane linkage, probably with the formation of various polyamines, and then the film degrades intensively between 150 to $305^{\circ} \mathrm{C}$. For the sample FR10, the degradation of the coating is significantly delayed, and it is believed that a gas phase flame retardant mechanism is the dominant effect as the coating is burning. But for FR50, it is concluded that an intumescent phosphorus-rich char layer leads to the improved fire resistance of the film.

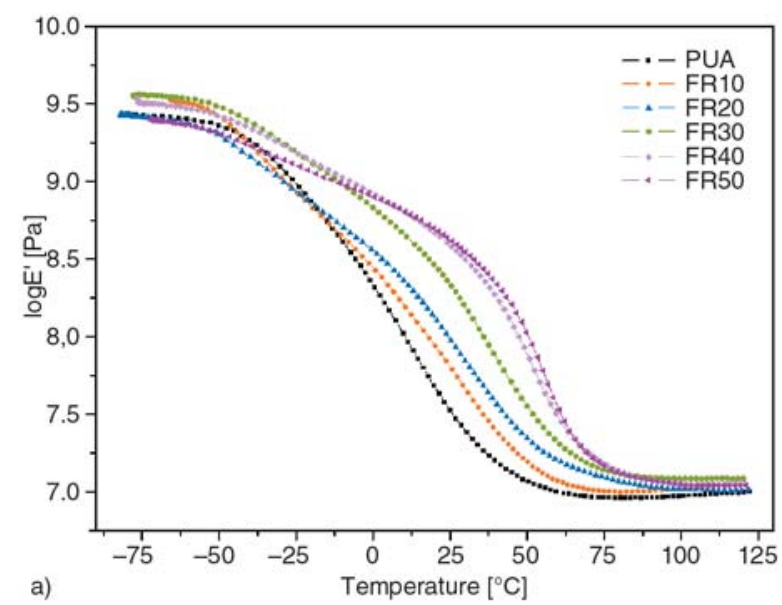

\subsection{Dynamic mechanical thermal behavior}

Dynamic mechanical thermal analysis (DMTA) was utilized to investigate the viscoelastic properties of the cured films in an effort to examine the microstructures of the polymer blends. The storage modulus reflects the elastic modulus of the coatings. The loss factor modulus is related to the energy lost due to the friction associated with the motion of polymer chains. The storage modulus $\left(E^{\prime}\right)$ and loss factor $(\tan \delta)$ curves of the pure PUA and its corresponding blends with N-PBAAP addition are shown in Figures 9a and 9b. The glass transition temperature $\left(T_{g}\right)$ is defined as the peak temperature of $\tan \delta$ curve. $T_{s}$ is the softening point defined as the extrapolated onset of the drop of storage modulus. Table 5 shows the data obtained from DMTA tests for all samples.

At the rubbery plateau $\left(T_{g}+40^{\circ} \mathrm{C}\right)$, the $E^{\prime}$ values for FR30 is measured to be $13.2 \mathrm{MPa}$, compared with $9.5 \mathrm{MPa}$ for that of pure PUA, almost an increase of $40 \%$ in storage modulus. However, as listed in Table 5, a decrease of $E^{\prime}$ values is observed with N-PBAAP content further increasing. The

Table 5. Rubbery storage modulus, $T_{s}, T_{g}$ and crosslink density (XLD) from DMTA

\begin{tabular}{|l|c|c|c|c|c|}
\hline $\begin{array}{c}\text { Sample } \\
\text { name }\end{array}$ & $\begin{array}{c}\mathbf{E}^{\prime} \\
{[\mathbf{M P a}]}\end{array}$ & $\mathbf{T}_{\mathbf{s}}\left[{ }^{\circ} \mathbf{C}\right]$ & $\mathbf{T}_{\mathbf{g}}\left[{ }^{\circ} \mathbf{C}\right]$ & $\mathbf{T}_{\mathbf{s}} / \mathbf{T}_{\mathbf{g}}$ & $\begin{array}{c}\mathbf{X L D} \\
{\left[\mathbf{m m o l} \cdot \mathbf{c m}^{-3}\right]}\end{array}$ \\
\hline PUA & 9.5 & -45.3 & 19.7 & 0.78 & 1.145 \\
\hline FR10 & 11.1 & -50.4 & 27.4 & 0.74 & 1.307 \\
\hline FR20 & 13.2 & -51.8 & 29.8 & 0.73 & 1.544 \\
\hline FR30 & 12.2 & -52.3 & 39.1 & 0.71 & 1.389 \\
\hline FR40 & 11.2 & -56.6 & 55.8 & 0.66 & 1.217 \\
\hline FR50 & 11.2 & -63.2 & 60.3 & 0.63 & 1.203 \\
\hline
\end{tabular}

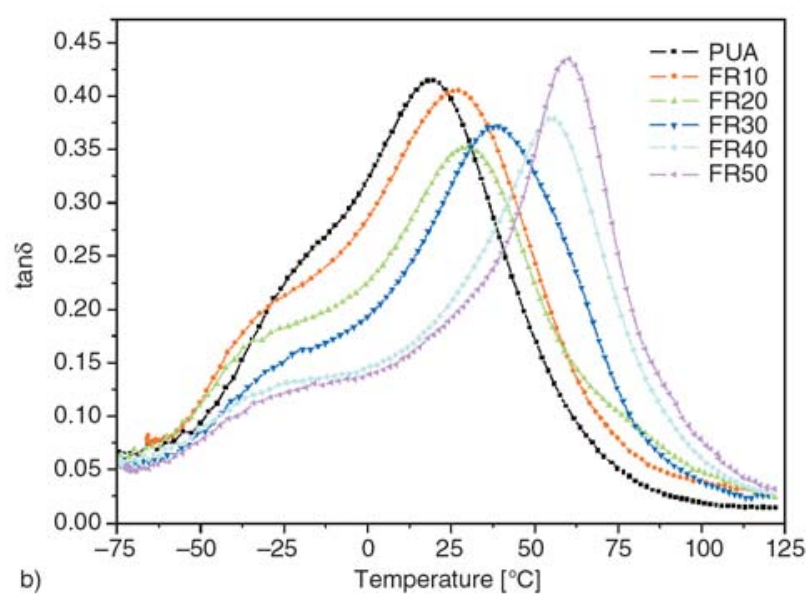

Figure 9. (a)The storage modulus $\left(E^{\prime}\right)$ curves of PUA, and PUA/N-PBAAP cured films. (b) The loss factor (tan $\delta$ ) curves of PUA, and PUA/N-PBAAP cured films 
variation of crosslink densities (XLD) of the samples shows the same trend as that of $E^{\prime}$.

These phenomena can be explained as follows. As far as the chemical structures of the two components are concerned, although both UA and N-PBAAP are difunctional oligomers i.e. diacrylates, N-PBAAP has a short molecular chain and low molecular weight compared with UA (Figure 1). As a result, the density of the acrylate group is higher for N-PBAAP than that of UA, which leads to the higher reactivity of N-PBAAP compared with UA oligomer. Therefore, as can be seen from Table 5, there is an increase in the glass transition temperature $\left(T_{g}\right)$ of the samples from 19.7 to $60.3^{\circ} \mathrm{C}$ with increasing N-PBAAP content, due to the rigid and short chain structure and higher density of acrylate group brought in the blends by N-PBAAP. However, in terms of $T_{s} / T_{g}$, which expresses the width of tan $\delta$ peak, things are different. It is a rule that a lower $T_{s} / T_{g}$ ratio leads to a wider $\tan \delta$ peak. The peak width broadens as the number of branching modes increases, which produces a wider distribution of structures. The range of temperatures at which the different network segments gain mobility therefore increases. From Table 5, the $T_{s} / T_{g}$ values of the samples decreased with increasing N-PBAAP content, indicating network heterogeneity increases of PUA/N-PBAAP films. According to the above, it can be concluded that the increasing of N-PBAAP content in the samples will at the same time bring in more functional groups and increase the network heterogeneity of the samples. Both of the two factors lead to the initial increase of the XLD and $E^{\prime}$ and then a decrease of them as N-PBAAP content increasing in the blends. Whatever, all the blends have a higher XLD than the pure PUA film.

In conclusion, the incorporation of N-PBAAP in the blends can obviously bring in more compactness and rigidity to the films, and increase the $T_{g}$ of the coatings. To some extent, it is important for practical application. Although the network heterogeneity increases slightly, it doesn't significantly affect the properties of the films.

\section{Conclusions}

A UV-reactive monomer N-PBAAP has been synthesized, and found effective for improving the flame retardancy of an ether-type PUA coating.
The MCC test revealed that the pHRR and HRC of the PUA coatings are reduced significantly in comparison with the pure PUA film. The THR values of all blends are lower than that of the pure PUA. The results suggested that N-PBAAP is an effective flame retardant for PUA coatings. The TG results showed that the PUA/N-PBAAP cured films have different degradation processes in air and in nitrogen when the N-PBAAP loading is low. And the char residues of the PUA coatings are obviously enhanced by the incorporation of N-PBAAP. The RT-FTIR analysis gave evidences for different flame retarding mechanisms of N-PBAAP when incorporated in the PUA films at different ratios. With $10 \mathrm{wt} \%$ of N-PBAAP loading in the coating formulation, probably a gas phase mechanism holds the dominant effect as the blend is burning. But when the content of N-PBAAP increases to $50 \mathrm{wt} \%$, a solid phase mechanism is proposed for the improved fire resistance of the film. According to the DMA analysis, it was found that the incorporation of N-PBAAP in the PUA films results in an increase of $T_{g}$ and a highest cross linking density for the sample containing $20 \mathrm{wt} \%$ of N-PBAAP.

\section{Acknowledgements}

The work was financially supported by the Program for Specialized Research Fund for the Doctoral Program of Higher Education (200803580008), the Program for Science and Technology of SuZhou (SG-0841), the Program for the graduate innovation fund in University of Science and Technology of China and the Opening Project of State Key Laboratory of Environmental Adaptability for Industrial Products.

\section{References}

[1] Roose P., Fallais I., Vandermiers C., Olivier M-G., Poelman M.: Radiation curing technology: An attractive technology for metal coating. Progress in Organic Coatings, 64, 163-170 (2009). DOI: $10.1016 /$ j.porgcoat.2008.08.020

[2] Masson F., Decker C., Jaworek T., Schwalm R.: UVradiation curing of waterbased urethane-acrylate coatings. Progress in Organic Coatings, 39, 115-126 (2000).

DOI: $10.1016 / \mathrm{S} 0300-9440(00) 00128-4$

[3] Uhl F. M., Davuluri S. P., Wong S-C., Webster D. C.: Organically modified montmorillonites in UV curable urethane acrylate films. Polymer, 45, 6175-6187 (2004).

DOI: $10.1016 /$ j.polymer.2004.07.001 
[4] Kim B. K., Ahn B. U., Lee M. H., Lee S. K.: Design and properties of UV cured polyurethane dispersions. Progress in Organic Coatings, 55, 194-200 (2006). DOI: 10.1016/j.porgcoat.2005.09.015

[5] Kim H., Urban M. W.: Molecular level chain scission mechanisms of epoxy and urethane polymeric films exposed to $\mathrm{UV} / \mathrm{H}_{2} \mathrm{O}$. Multidimensional spectroscopic studies. Langmuir, 16, 5382-5390 (2000). DOI: $10.1021 / \mathrm{la} 990619 \mathrm{i}$

[6] Kumar V., Bhardwaj Y. K., Goel N. K., Francis S., Dubey K. A., Chaudhari C. V.: Coating characteristics of electron beam cured Bisphenol A diglycidyl ether diacrylate-co-aliphatic urethane diacrylate resins. Surface and Coatings Technology, 202, 5202-5209 (2008).

DOI: $10.1016 /$ j.surfcoat.2008.06.047

[7] Yeh J-T., Shu Y-C.: Characteristics of the degradation and improvement of the thermal stability of poly(siloxane urethane) copolymers. Journal of Applied Polymer Science, 115, 2616-2628 (2010).

DOI: $10.1002 / a p p .29023$

[8] Yen F. S., Lin L. L., Hong J. L.: Hydrogen-bond interactions between urethane-urethane and urethane-ester linkages in a liquid crystalline poly(ester-urethane). Macromolecules, 32, 3068-3079 (1999). DOI: $\underline{10.1021 / \mathrm{ma} 9804186}$

[9] Chattopadhyay D. K., Raju K. V. S. N.: Structural engineering of polyurethane coatings for high performance applications. Progress in Polymer Science, 32, 352-418 (2007). DOI: 10.1016/j.progpolymsci.2006.05.003

[10] Lu W. H., Xu W. J., Wu Y. M., Zhou X., Lu Y. B., Xiong Y. Q.: Synthesis of dendritic poly(urethane acrylate) used for UV-curable coatings. Progress in Organic Coatings, 56, 252-255 (2006).

DOI: 10.1016/j.porgcoat.2005.10.004

[11] Decker C., Zahouily K.: Photodegradation and photooxidation of thermoset and UV-cured acrylate polymers. Polymer Degradation and Stability, 64, 293-304 (1999).

DOI: 10.1016/S0141-3910(98)00205-5

[12] Xing W. Y., Hu Y., Song L., Chen X. L., Zhang P., Ni J. X.: Thermal degradation and combustion of a novel UV curable coating containing phosphorus. Polymer Degradation and Stability, 94, 1176-1182 (2009). DOI: 10.1016/j.polymdegradstab.2009.02.014

[13] Jimenez M., Duquesne S., Bourbigot S.: Intumescent fire protective coating: Toward a better understanding of their mechanism of action. Thermochimica Acta, 449, 16-26 (2006). DOI: $\underline{10.1016 / j . t c a .2006 .07 .008}$

[14] Gu J-W., Zhang G-C., Dong S-L., Zhang Q-Y., Kong J.: Study on preparation and fire-retardant mechanism analysis of intumescent flame-retardant coatings. Surface and Coatings Technology, 201, 7835-7841 (2007).

DOI: $\underline{10.1016 / \text { j.surfcoat.2007.03.020 }}$
[15] Chen X. L., Hu Y., Jiao C. M., Song L.: Preparation and thermal properties of a novel flame-retardant coating. Polymer Degradation and Stability, 92, 11411150 (2007).

DOI: 10.1016/j.polymdegradstab.2007.01.031

[16] Zhu S. W., Shi W. F.: Thermal degradation of a new flame retardant phosphate methacrylate polymer. Polymer Degradation and Stability, 80, 217-222 (2003). DOI: $10.1016 / \mathrm{S} 0141-3910(02) 00401-9$

[17] Fang Z. P., Guo F., Tong L. F.: Effect of a novel phosphorous-nitrogen containing intumescent flame retardant on the fire retardancy and the thermal behaviour of poly(butylene terephthalate). Polymer Degradation and Stability, 91, 1295-1299 (2006).

DOI: $10.1016 /$ j.polymdegradstab.2005.08.013

[18] Nguyen C., Kim J.: Thermal stabilities and flame retardancies of nitrogen-phosphorus flame retardants based on bisphosphoramidates. Polymer Degradation and Stability, 93, 1037-1043 (2008).

DOI: $10.1016 /$ j.polymdegradstab.2008.03.024

[19] Lyon R. E., Walters R. N., Stoliarov S. I.: Screening flame retardants for plastics using microscale combustion calorimetry. Polymer Engineering and Science, 47, 1501-1510 (2007).

DOI: $10.1002 /$ pen.20871

[20] Lyon R. E.: Heat release kinetics. Fire and Materials, 24, 179-186 (2000).

DOI: 10.1002/1099-1018(200007/08)24:4<179::AIDFAM736>3.0.CO;2-V

[21] Lin M-F., Tsen W-C., Shu Y-C., Chung F-S.: Effect of silicon and phosphorus on the degradation of polyurethanes. Journal of Applied Polymer Science, 79, 881-899 (2001).

DOI: $10.1002 / 1097-4628(20010131) 79: 5<881::$ AID APP130>3.0.CO;2-I

[22] Chuang F. S., Tsen W. C., Shu Y. C.: The effect of different siloxane chain-extenders on the thermal degradation and stability of segmented polyurethanes. Polymer Degradation and Stability, 84, 69-77 (2004).

DOI: 10.1016/j.polymdegradstab.2003.10.002

[23] Matsunaga K., Tajima M., Yoshida Y.: Thermal degradation of carboxylate-based polyurethane anionomers. Journal of Applied Polymer Science, 101, 573-579 (2006).

DOI: $10.1002 / a p p .23574$

[24] Duquesne S., Bras M., Bourbigot S., Delobel R., Camino G., Eling B., Lindsay C., Roels T., Vezin H.: Mechanism of fire retardancy of polyurethanes using ammonium polyphosphate. Journal of Applied Polymer Science, 82, 3262-3274 (2001).

[25] Wang T-L., Cho Y-L., Kuo P-L.: Flame-retarding materials. II. Synthesis and flame-retarding properties of phosphorus-on-pendent and phosphorus-on-skeleton polyols and the corresponding polyurethanes. Journal of Applied Polymer Science, 82, 343-357 (2001).

DOI: $\underline{10.1002 / a p p .1858}$ 
[26] Wang G. A., Cheng W. M., Tu Y. L., Wang C. C., Chen C. Y.: Characterizations of a new flame-retardant polymer. Polymer Degradation and Stability, 91, 3344-3353 (2006).

DOI: $\underline{10.1016 / j . p o l y m d e g r a d s t a b .2006 .06 .001}$

[27] Hsiue G-H., Shiao S-J., Wei H-F., Kuo W-J., Sha YA.: Novel phosphorus-containing dicyclopentadienemodified phenolic resins for flame-retardancy applications. Journal of Applied Polymer Science, 79, 342349 (2001).

DOI: $10.1002 / 1097-4628(20010110) 79: 2<342:: A I D-$ APP180>3.0.CO;2-8
[28] Setnescu R., Jipa S., Setnescu T., Kappel W., Kobayashi S., Osawa Z.: IR and X-ray characterization of the ferromagnetic phase of pyrolysed polyacrylonitrile. Carbon, 37, 1-6 (1999).

DOI: 10.1016/S0008-6223(98)00168-7

[29] Bugajny M., Bourbigot S., Bras M., Delobel R.: The origin and nature of flame retardance in ethylene-vinyl acetate copolymers containing hostaflam AP 750 . Polymer International, 48, 264-270 (1999).

DOI: 10.1002/(SICI)1097-0126(199904)48:4<264:: $\underline{\text { AID-PI118>3.0.CO;2-M }}$ 